\title{
APPLICATION OF ORP FOR THE EVALUATION OF WATER CONTAMINATION
}

\author{
Viktoras Račys \\ Linas Kliučininkas \\ Dalia Jankūnaitè \\ Kaunas University of Technology, Lithuania \\ Ramunè Albrektiene \\ Vilnius Gediminas Technical University, Lithuania
}

\begin{abstract}
ABATRACT
Water pollution characterization consumes a lot time, facilities and materials. Application of easy applicable and accurate method for pollution determination is quiet actual still now. Redox reactions in water are usually biologically mediated and therefore, the oxidation reduction potential (ORP) of wastewater and groundwater systems depends upon biodegradation processes. Oxidation and reduction reactions control the behaviour of many chemical constituents in the water. The determination of ORP is advisable in water that contains a relatively high concentration of a redox-active species, e.g., organic carbon, the salts of many metals $\left(\mathrm{Fe}^{2+}, \mathrm{Mn}^{2+}\right)$ and strong oxidising (chlorine, oxygen) and reducing (sulfite ion) agents. Water pollution characterization upon ORP and different types of polluted water was investigated. The main outcome of this research - ORP can be used as easy applicable measurement for different types of water as pollution indicator.
\end{abstract}

\section{KEYWORDS}

Water pollution; Waste water; Ground water; Oxidation reduction potential; Iron; Manganese

\section{INTRODUCTION}

The oxidation/reduction potential (ORP) is a measure of electron activity and is an indicator of the relative tendency of a solution to accept or transfer electrons. Oxidation and reduction (redox) reactions control the behaviour of many chemical constituents in the natural waters and sewage. The reactivity and solubility of critical elements in living systems is strongly dependent on redox conditions. ORP values are affected by all oxidising and reducing agents, not just acids and bases. High $\mathrm{pH}$ ionized water demonstrates a negative ORP and so is a reducing agent or "antioxidant".

The most important redox reactions in natural waters are the oxidation of organic matter and the corresponding reduction reactions: reduction of oxygen to water, nitrate to elementary nitrogen $\mathrm{N}_{2}$, manganese (III/IV) to Mn (II), and iron (III) to Fe (II), sulphate to sulphide and $\mathrm{CO}_{2}$ to methane.

Many parameters are used to determine wastewater treatment performance, including dissolved oxygen (DO), $\mathrm{pH}$, total suspended solids (TSS) and biochemical oxygen demand (BOD). Additional parameters may help with understanding the potential for improving 
system operation. ORP has its applications in industries like metal finishing, bleach production, poultry processing, fruit and vegetable washing, pulp bleaching in paper industry.

\section{EXPERRIMENTAL PROCEDURES}

During the main sampling campaign samples were collected for chemical analysis of BOD, $\mathrm{Fe}, \mathrm{Mn}$, and As. The procedures of water sampling, preservation, transportation and were carried out according to the procedures set in ISO 5667-3:2006 standard [1]. The concentration of BOD were measured according to the method, set in the international standard ISO 5815-1:2003. The concentrations of Fe and Mn during this campaign were measured according to the method, set in the international standard ISO 8288:1986, based on the atomic absorption measurement and adapted for $\mathrm{Fe}$ and $\mathrm{Mn}$ determination [2]. The concentration of As was measured according to the international standard ISO 11969:1996, using method, based on the atomic absorption measurement of arsenic generated by the thermal decomposition of arsenic (II) hydride [3]. These analyses were performed using AA6800 (Shimadzu Corp., Japan) spectrophotometer.

All the analyseswere made in accordance with the requirements for the purity of reagents, their preparation and storage listed in referred international standards with suitable laboratory devices and following the requirements for the analyses and presentation of results. Total content (soluble, insoluble and organic) of all the measured metals was determined in every case.

The oxidation-reduction potential (ORP, $\mathrm{mV}$ ) measurements were done on site, using WTW $\mathrm{pH} /$ Cond 340i portative meter with exchangeable electrodes for ORP (WTW SenTix Electrode ORP) measurement. Sampling for on site measurements was carried out according to the standard procedures, setting principles for design of sampling programmes and techniques (ISO 5667-1:2006); sampling from piped distribution systems (ISO 5667-5:2006); sampling and handling of water samples taken from groundwater (ISO 5667-11:2009) [4, 5, $6]$.

\section{RESULTS AND DISSCUSION}

\subsection{Case study I: ORP and surface water infiltration into groundwater}

Number of authors have confirmed the direct dependence on reductive conditions to the increase in concentration of manganese in groundwater by claiming that the reductive conditions affect the release of manganese from the aquifer rocks. Our investigation revealed that $\mathrm{Mn}$ is behaving differently from two over measured metals - Fe and $\mathrm{Mn}$ (see Figure 1). In lower ORP values As and Fe concentration is increasing and $\mathrm{Mn}$ concentration is decreasing. It can be suggested, that manganese comes from another source than aquifer minerals, one of the possible sources - leaching of Mn salts, deposited in the sedimentary layer of the Reservoir. This partially confirms the impact of the distance from the Reservoir on manganese concentrations.

From the results obtained, it can be concluded that in the reductive environment iron and arsenic dissolution is parallel. Previous authors have already noted that arsenic concentration in groundwater has been limited at higher ORP values. With decreasing ORP an increase in arsenic concentrations has been recorded, influenced by the reductive dissolution of iron oxyhydroxides [7, 8]. It is known that both Fe (II) and As (III) are behaving similarly in the similar reductive conditions [7]. 


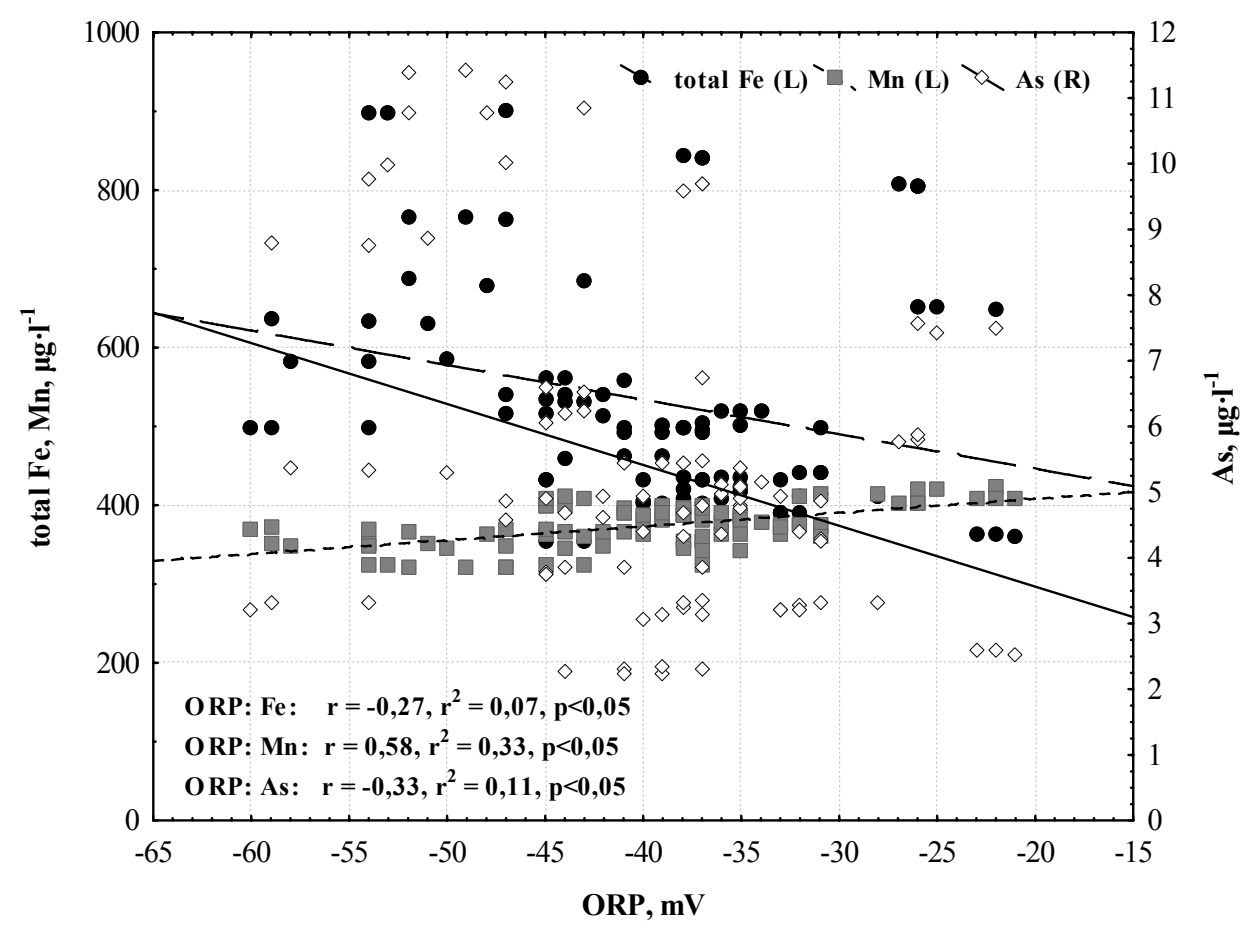

Figure 1. Dependence of total Fe, Mn and As on ORP in groundwater of Petrasiunai wellfield

The natural arsenic contamination, as well as in the case of iron and manganese, is affected by a whole range of (bio)geochemical processes that determine the nature and mobility of arsenic under various reductive conditions [8]. The main processes, determining the access of arsenic into the water are associated with reductive dissolution of arsenic-containing minerals (e.g., arsenopyrite), or iron and manganese oxyhydroxides as well as other iron and manganese compounds with adsorbed arsenic [7, 8, 9]. Such dissolution is possibly related to the spatial and seasonal changes in the reductive status of the infiltration zone due to the fluctuations of groundwater level and changes in the activity of microorganisms [8]. Therefore, in the highly arsenic-contaminated groundwater, large concentrations of iron are often found [7].

\subsection{Case study II: ORP in waste water treatment process optimisation}

Many parameters are used to determine wastewater treatment performance, including dissolved oxygen (DO), $\mathrm{pH}$, total suspended solids (TSS) and biochemical oxygen demand (BOD). Additional parameters may help with understanding the potential for improving system operation. One such parameter is the oxidation-reduction potential (ORP) $[10,11,12]$.

BOD is an important parameter for the biological wastewater treatment process and may influence effectiveness of $\mathrm{N}$ and $\mathrm{P}$ removal. Analysis of BOD is time consuming procedure alternatively ORP measurements could be used for more effective regulation of wastewater treatment plant operation. In our research among others relationship between BOD and ORP at Kaunas WWTP was investigated. As both parameters depend on wastewater flow most likely they are relative. 


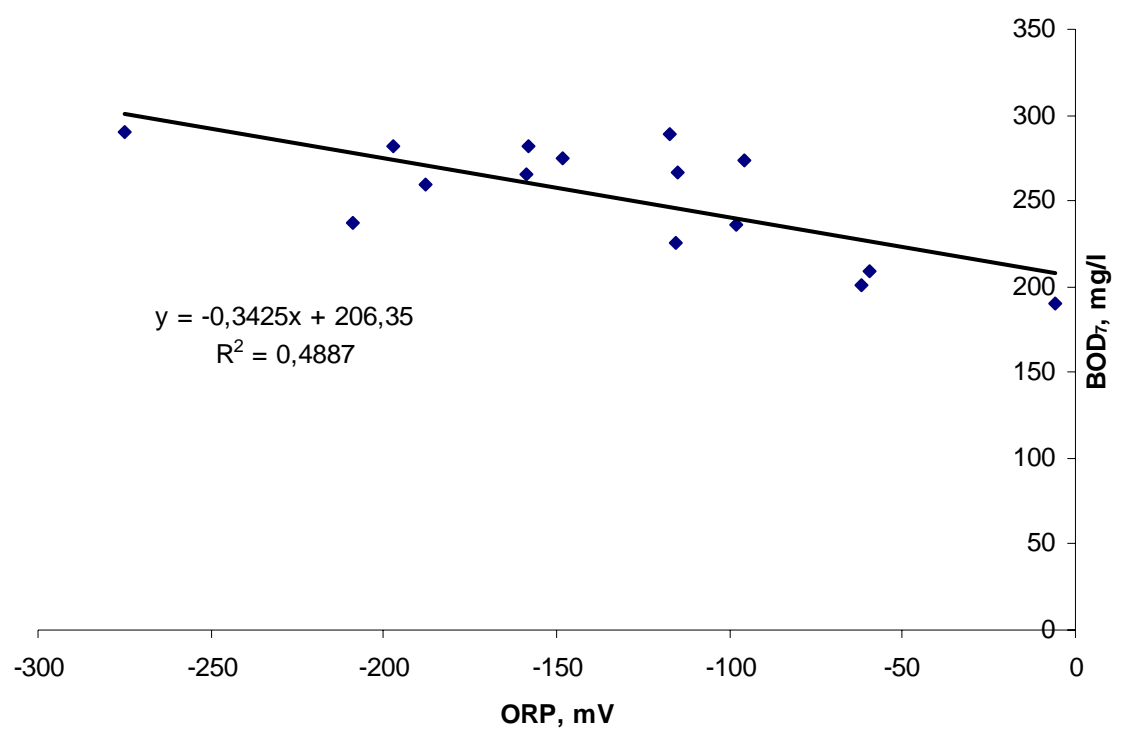

Figure 2. Correlation of ORP with $\mathrm{BOD}_{7}$ in wastewater

BOD of wastewater after mechanical treatment process in most of the cases ranged $260-300$ $\mathrm{mgO}_{2} / 1$ while ORP varied between -200 and $-100 \mathrm{mV}$. Correlation coefficient $-0.7(\mathrm{p}=0.0039)$ shows strong relationship between those parameters. Relation between ORP and BOD is presented in Figure 2. Increase of BOD concentration in wastewater results in lower values of oxidation - reduction potential, reductive conditions are formed.

\subsection{Case study III: ORP in groundwater quality characterisation}

Redox reactions in groundwater are usually biologically mediated and therefore, the redox potential of a groundwater system depends upon and influences rates of biodegradation. Knowledge of the redox potential of groundwater is also important because some biological processes only operate within a prescribed range of redox conditions. Knowledge of the redox potential of groundwater can be used as an indicator of certain geochemical activities such as sulphate reduction. The redox potential of groundwater generally ranges from $-400 \mathrm{mV}$ to $800 \mathrm{mV}$.

The determination of ORP is particularly worthwhile in water that contains a relatively high concentration of a redox-active species, e.g., the salts of many metals $\left(\mathrm{Fe}^{2+}, \mathrm{Fe}^{3+}\right)$ and strong oxidising (chlorine) and reducing (sulphite ion) agents. Thus, ORP can sometimes be utilised to track the metallic pollution of groundwater. However, ORP is a non-specific measurement - that is, the measured potential is reflective of a combination of the effects of all the dissolved species in the medium. Because of this factor, the measurement of ORP in relatively clean environmental water (ground, surface, estuarine, and marine) has only limited utility unless a predominant redox-active species is known to be present. Care is required not to "over-interpret" ORP data unless specific information about the site is known $[13,14]$.

About $87 \%$ of the investigated groundwater bodies and about $55 \%$ of groundwater wells in Lithuania (see Figure 3) used for potable water do not meet requirements of the iron concentration in water $(\geq 0.2 \mathrm{mg} / \mathrm{l})$. 

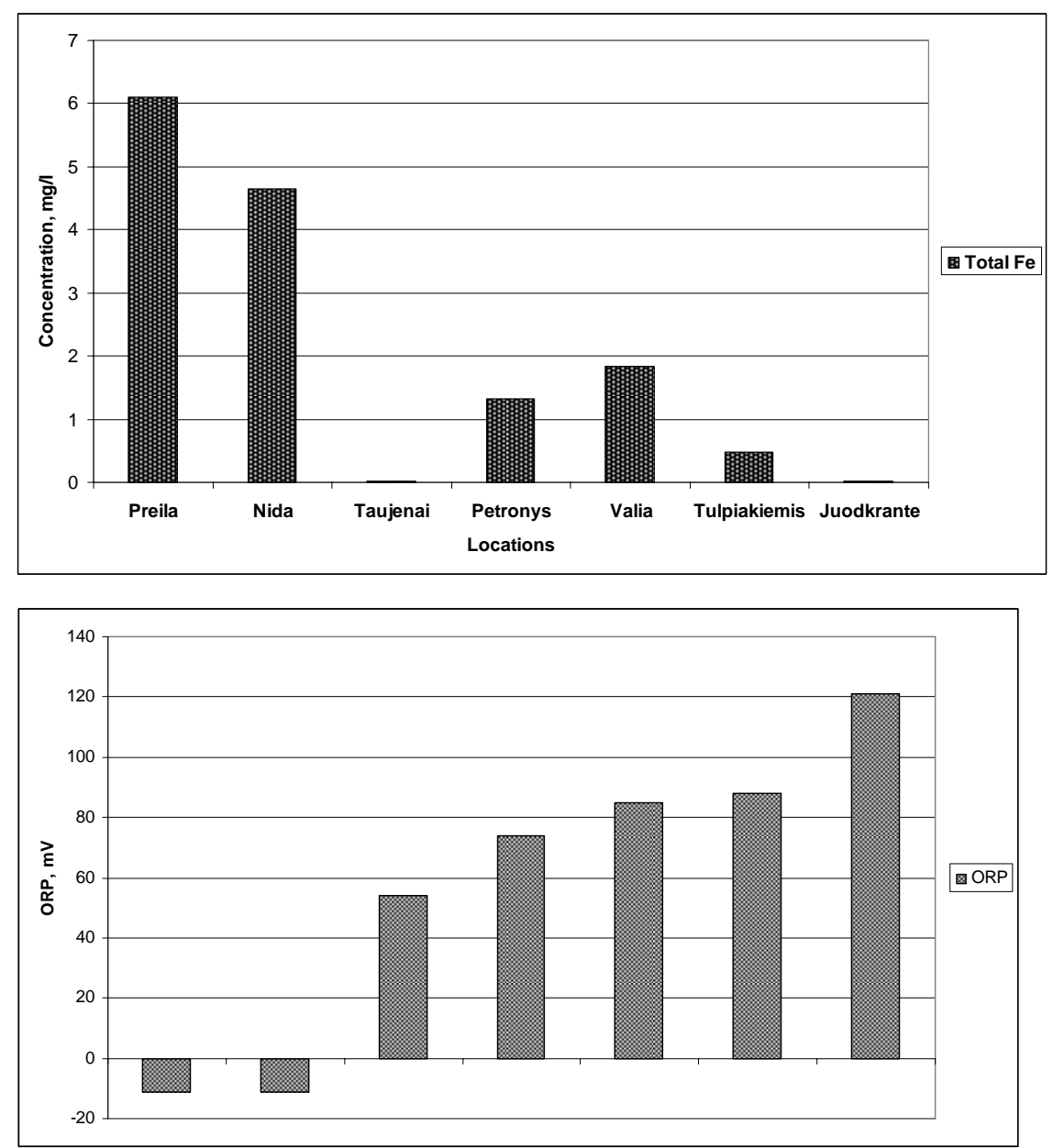

Figure 3. Concentration of total iron ( $\mathrm{Fe}$ ) and oxidation reduction potential (ORP) in ground water wells at different locations

The determination of ORP is particularly worthwhile in water that contains a relatively high concentration of a redox-active species, e.g., the salts of many metals $\left(\mathrm{Fe}^{2+}, \mathrm{Fe}^{3+}\right)$ and strong oxidising (chlorine) and reducing (sulphite ion) agents. Thus, ORP can sometimes be utilised to track the metallic pollution of groundwater [15].

This chapter provides results of the study, where oxidation reduction potential (ORP) was used as an indicator to track trends of the metallic pollution of the drinking ground water. More than $50 \%$ of groundwater wells in the study area used for potable water do not meet requirements of the iron concentration in water $(\geq 0.2 \mathrm{mg} / \mathrm{l})$. Water wells with very high, medium and low concentrations of iron were selected for detail analysis. Measurements were performed at Barzdai and Keturnaujiena settlements (water consumption less than 100 $\mathrm{m}^{3} /$ day). In 2010 authors have performed several sampling campaigns in drinking ground water wells (see Fig. 4), also tap water samples were taken in the selected residences. 

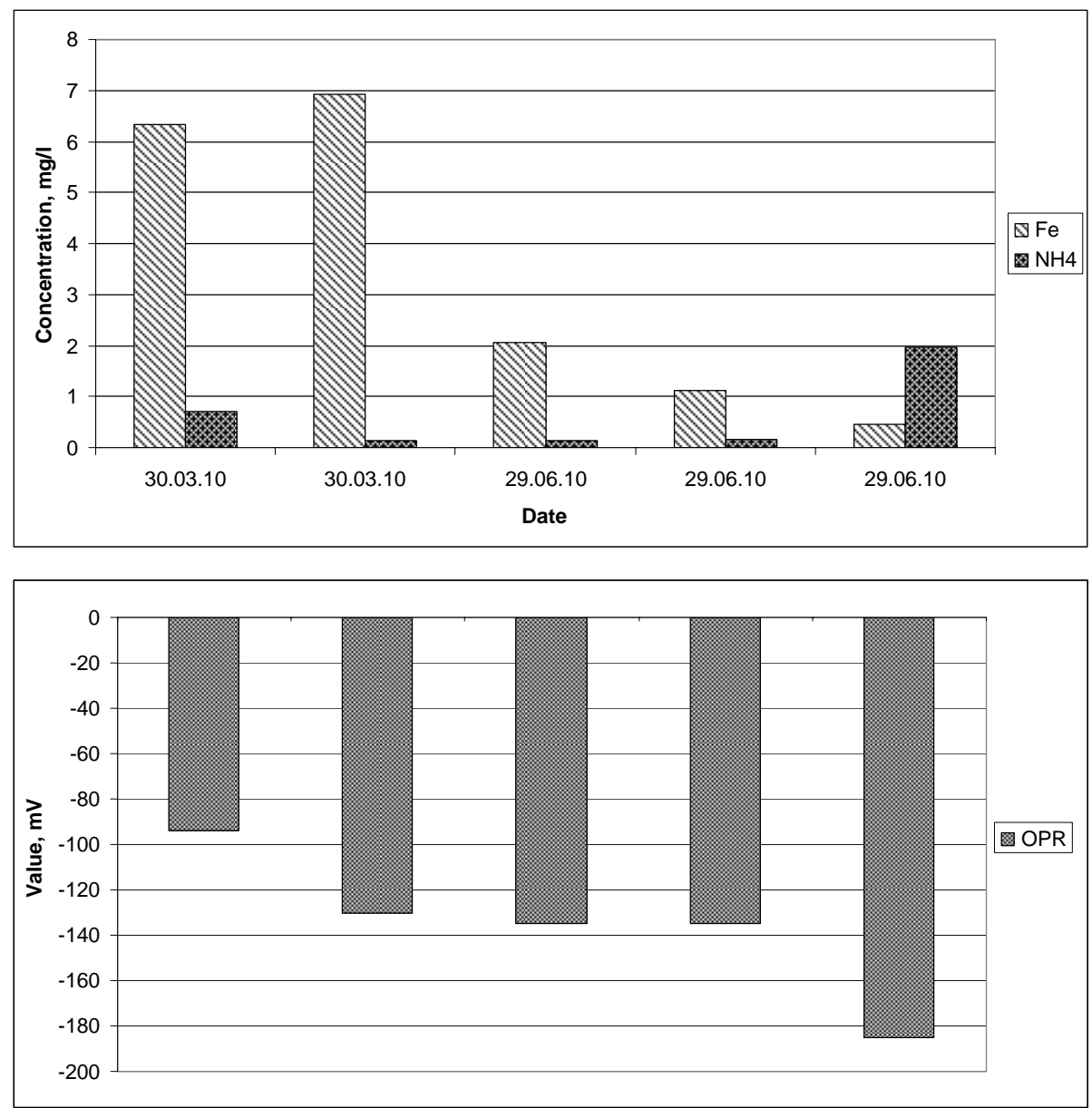

Figure 4. Concentration of total iron ( $\mathrm{Fe}$ ), ammonium (NH4) and oxidation reduction potential (ORP) in ground water well at Barzdai settlement

The maximal concentration of total iron $6.93 \mathrm{mg} / 1$ was indicated in water well at Barzdai settlement, respectively minimal value $0.065 \mathrm{mg} / \mathrm{l}$ was registered at Keturnaujiena settlement. The concentrations of $\mathrm{NH}_{4}^{+}$in Barzdai water well have varied from $0.14 \mathrm{mg} / 1$ in June to 0.72 $\mathrm{mg} / \mathrm{l}$ in March; the respective ORP values varied from $-94 \mathrm{mV}$ in March to $-130 \mathrm{mV}$ in June, 2010. The analysis results show high correlation between $\mathrm{NH}_{4}{ }^{+}$and ORP values, however there were not noticed significant correlations between concentrations of total iron and ORP.

Comparative analyses of tap water in the water supply system (without devices for iron and manganese removal) have showed that in residences, where water was supplied from the water well with very high concentration of iron, considerable decrease of iron concentration (in average by 3 times). In the residences, where water was supplied from the water well with high concentration of iron, the concentration remained unchanged. Tap water at the residences with low concentration of iron in water well has indicated the increase of the iron concentration. In general, analyses of tap water have showed high correlation between concentration of iron and ORP, also some regularity was observed between concentrations of iron an distance in the water supply system from the water well.

\section{CONCLUSIONS}


The oxidation/reduction potential (ORP) is an integrative indicator, which defines certain geochemical processes in the surface water infiltration into the groundwater (Case study I). ORP monitoring data could be used to characterise trends of natural geochemical processes.

Results of the Case study II demonstrated relationship between BOD of wastewater after mechanical treatment process and ORP. Waste water that contains a relatively high concentration of nitrogen and phosphorus requires precise technological operation and maintenance. Further studies of ORP determination in waste water treatment process are recommended.

Results of the Case study III show that ORP monitoring data could be used to track conditions and seasonal variations of metallic pollution of groundwater and could provide feedback for more efficient metal removal process from the drinking ground water.

The techniques for removing iron and manganese from water are based on the oxidation of relatively soluble $\mathrm{Fe}(\mathrm{II})$ and $\mathrm{Mn}$ (II) to the insoluble $\mathrm{Fe}$ (III) and $\mathrm{Mn}$ (III, IV) and the oxidation of any organic-complex compounds, thus, on-line monitoring of redox potential could provide feedback in the oxidation process adjustment to make metal remove more efficient.

\section{ACKNOWLEDGEMENTS}

This study was supported by the project From theory and plans to eco-efficient and sustainable practices to improve the status of the Baltic Sea, WATERPRAXIS (Grant Contract \# 010) of the Baltic Sea Region Programme 2007-2013.

\section{REFERENCES}

[1] ISO 5667-3:2003 Water quality - Sampling - Part 3: Guidance on the preservation and handling of water samples.

[2] ISO 8288:1986 Water quality - Determination of cobalt, nickel, copper, zinc, cadmium and lead - Flame absorption spectrometric methods.

[3] ISO 11969:1996 Water quality - Determination of arsenic - Atomic absorption spectrometric method (hydride technique).

[4] ISO 5667-1:2006 Water quality - Sampling - Part 1: Guidance on the design of sampling programmes and sampling techniques.

[5] ISO 5667-5:2006 Water quality - Sampling - Part 5: Guidance on sampling of drinking water from treatment works and piped distribution systems.

[6] ISO 5667-11:2009 Water quality - Sampling - Part 11: Guidance on sampling of groundwaters.

[7] Bose, P., Sharma, A., 2002. Role of iron in controlling speciation and mobilization of arsenic in subsurface environment. Water Research. 36, 4915-4926.

[8] Drahota, P., Rohovec, J., Filippi, M., Mihaljevič, M., Rychlovsky, P., Červeny, V., Pertold, Z., 2009. Mineralogical and geochemical controls of arsenic speciation and mobility under different redox conditions in soil, sediment and water at the Mokrsko-West gold deposit, Czech Republic. Science of the Total Environment. 407, 3372-3384.

[9] Zahid, A., Hassan, M.Q., Breit, G.N., Balke K.-D., Flegr M., 2009. Accumulation of iron and arsenic in the Chandina alluvium of the lower delta plain, Southeastern Bangladesh. Environmental Geochemistry and Health. 31, 69-84. 
[10] Pankaj, T., Tapas, N., Pallavi, U., Pravin, M., 2008. Correlating on-line monitoring parameters, $\mathrm{pH}, \mathrm{DO}$ and ORP with nutrient removal in an intermittent cyclic process bioreactor system. Bioresource Technology, 99(16), 7630-7635.

[11] Kishida, N., Kim, J.-H., Chen, M., Sasaki, H., Sudo, R., 2003. Effectiveness of oxidation-reduction potential and $\mathrm{pH}$ as monitoring and control parameters for nitrogen removal in swine wastewater treatment by sequencing batch reactors. Journal of Bioscience and Bioengineering, 96(3), 285-290.

[12] Yu, R.-F., Chen, H.-W., Cheng, W.-P., Shen, Y.-C., 2008. Dynamic control of disinfection for wastewater reuse applying $\mathrm{ORP} / \mathrm{pH}$ monitoring and artificial neural networks. Original Research Article Resources, Conservation and Recycling, 52(8-9), 1015-1021.

[13] Weng, H.-X., Qin, Y.-CH., Chen, X.-H., 2007. Elevated iron and manganese concentrations in groundwater derived from the Holocene transgression in the Hang-JiaHu Plain, China. Hydrology Journal, 15, 715-726.

[14] Tremblay, C. V., Beaubien, A., Charles, P., Nicell, J.A., 1998. Control of biological iron removal from drinking water using oxidation-reduction potential. Water Science and Technology, 38(6), 121-128.

[15] Diliūnas, J., Jurevičius, A., Zuzevičius, A., 2006. Formation of iron compounds in the Quarternary groundwater of Lithuania. Geologija, 55, 67-74. 